\title{
Octreotide scintigraphy localizes somatostatin receptor-positive islet cell carcinomas
}

\author{
W. Becker, J. Marienhagen, R. Scheubel, A. Saptogino, W.H. Bakker, W.A.P. Breeman, and F. Wolf \\ Department of Nuclear Medicine and Internal Medicine I, University of Erlangen-Nürnberg, FRG, and \\ Department of Nuclear Medicine, University of Rotterdam, The Netherlands
}

Received 10 May and in revised form 1 June 1991

\begin{abstract}
Tyr-3-Octreotide is a synthetic derivative of somatostatin and a somatostatin-receptor analogue. The iodine-123-labelled compound localizes somatostatin-receptor-positive tumours. In this paper two patients are reported in whom somatostatin receptors were demonstrated in vitro. In a 60-year-old female with an islet cell carcinoma of the pancreas, multiple liver metastases and previously unrecognized bone metastases in the right acetabulum could be diagnosed as the reason for a persistent hypoglycaemia. In a 60-year-old male an islet cell carcinoma of the pancreas was localized with ${ }^{123}$ I-Tyr-3-octreotide. The somatostatin receptors were demonstrated in vitro and the tumour was successfully treated with somatostatin. These studies demonstrate that ${ }^{123} \mathrm{I}$-Tyr-3-octreotide offers the possibility of localizing somatostatin-receptor-positive tumours and their metastases. Moreover the method makes it possible to determine the receptor status of a tumour in vivo.
\end{abstract}

Key words: Iodine-123-Tyr-Octreotide - Somatostatin receptors - In vivo - In vitro - Pancreatic islet cell carcinoma

Eur J Nucl Med (1991) 18:924-927

\section{Introduction}

Iodine 123 tyr-3-octreotide 201-995, a synthetic derivative of somatostatin, is a somatostatin receptor analogue (Bakker et al. 1990) which has been introduced for the localization of somatostatin receptor-positive tumours (Kremning et al. 1989; Lamberts et al. 1990a, b, c). A parallel between the evidence of somatostatin receptors on tumours and in vivo and in vitro effects of octreotide on hormonal release from these tumours indicates that a positive scan predicts a good suppressive effect of octreotide on hormonal hypersecretion by these endocrine tumours. This is one of the possible future indications for an iodine 123 tyr-3-octreotide scan. Another indication is based on the fact that, with the exception of insulinomas, most endocrine pancreatic tumours are malignant and have already metastasized at the time of diagnosis (Bloom and Polak 1980; Stefani et al. 1974), so surgery is considered to be the primary treatment. For this reason, a specific and sensitive method for the localization of all tumour manifestations is required.

This case report confirms the positive result of previous papers dealing with iodine 123 tyr-3-octreotide (Krenning et al. 1989; Lamberts et al. 1990a, b, c). Nontumour related uptake is also described.

\section{Materials and patients}

Radiopharmaceuticals. The radiolabelling of tyr-3-octreotide was performed according to a previously published method (Bakker et al. 1990). Tyr-3-octreotide was obtained from Sandoz (Basel, Switzerland). The radioiodination of the tracer was performed with ${ }^{123}$ I from Medgenix, Belgium. The radioactivity concentration and specific activities amounted to $3.7 \mathrm{GBq} / \mathrm{ml}$. Normal patient activity was $185 \mathrm{MBq}$ iodine 123 tyr-3-octreotide. There were no side effects recorded. All patients gave their written informed consent for this examination.

Data acquisition. Gamma-camera images were performed as planar $(128 \times 128$ matrix $)$ and SPET images $(64 \times 64$ matrix $)$ with a Sicmens Rota gamma-camera fitted with a low-energy, high-resolution collimator. Data were collected with a Siemens Microdelta computer. The image time was routinely 5-45 min post injection (p.i.) and 2-3 h p.i. The relevant images were performed during the first $30 \mathrm{~min}$, before the beginning of the intestinal excretion of the ${ }^{123}$ I-labelled metabolites of tyr-3-octreotide. The SPET image of the liver was performed $2 \mathrm{~h}$ p.i. after significant clearance of the iodine by the liver.

In vitro determination of somatostatin receptors. Somatostatin receptors were measured by autoradiography on cryostat sections of the tumour tissues as described previously in detail for various endocrine tumours (Reubi et al. 1987, 1990).

Case reports

Patient 1. A 60 -year-old man who suffered from a weight loss of $10 \mathrm{~kg}$ and heavy diarrhoea during the past 2 months was admit- 


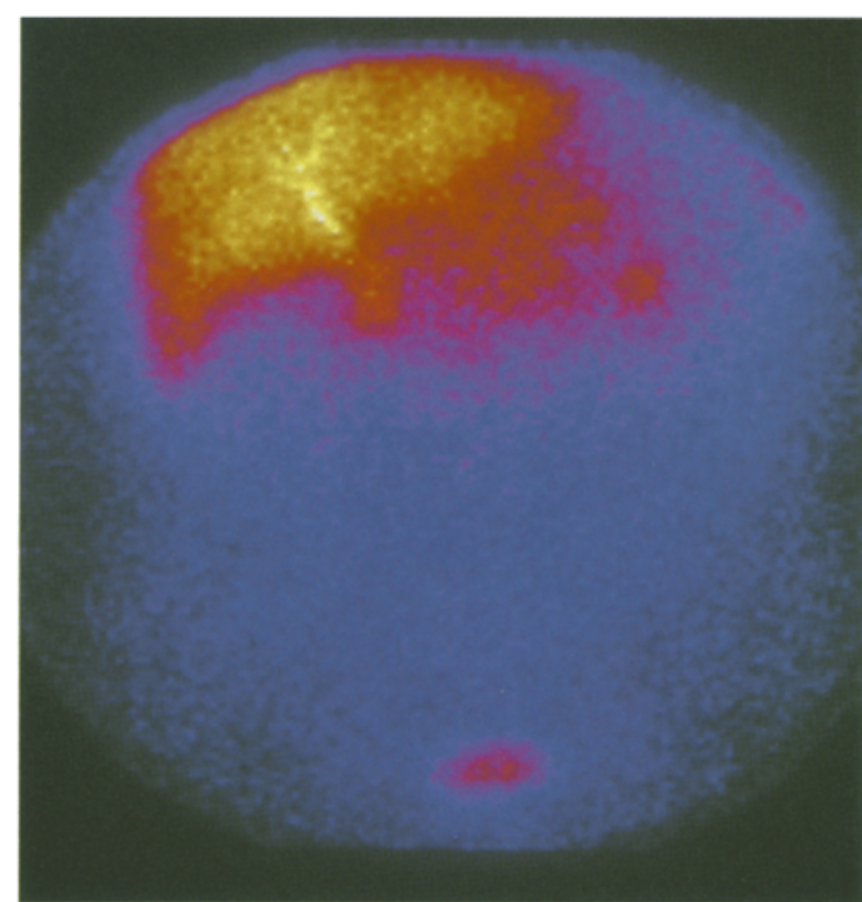

Fig. 1. A 60-year-old man with somatostatin receptor-positive, non-resectable pancreatic islet carcinoma, which is specifically shown with iodine 123 tyr-3-octreotide between the activity of both kidneys in a planar abdominal scan

ted to our hospital (April 1984). Ultrasound, computed X-ray tomography and magnetic resonance imaging localised a pancreatic mass of $9 \times 6 \mathrm{~cm}$ diameter with compression of the vena cava and aorta without any signs of infiltration but with occlusion of the vena lienalis and the vena mesenterica superior. The tumour of the pancreas produced no clinically detectable product and was judged to be inactive.

During explorative surgery the tumour proved not to be resectable (May 1984). Biopsy specimen of the tumour revealed an islet cell carcinoma. At that time the autoradiography of somatostatin receptors was not yet possible, and Sandostatin was not available. The patient underwent 21 courses of fluorouracil and streptozocin treatment over the subsequent 3 years. The tumour size did not change, but the symptoms, especially weakness and faintness, increased. At this time (Nov. 1987) an ultrasound-guided biopsy of the pancreatic tumour was examined with autoradiography, which demonstrated a significant number of somatostatin receptors expressed on the tumour tissue. Following this diagnosis treatment with $3 \times 500 \mu \mathrm{g}$ of Sandostatin given subcutaneously was started. From this time on, the patient recovered quickly, and the tumour size decreased slightly. In Aug. 1990 an iodine 123 tyr-3-octreotide scan was performed after a 3-day interruption of the Sandostatin treatment. The scan (Fig. 1) demonstrated a large tumour mass between both kidneys and under the liver with significant tracer uptake. Other manifestations of the tumour could not be detected.

Patient 2. A 60-year-old woman was admitted to our hospital (April 1988) with hypoglycaemia, weight loss of $20 \mathrm{~kg}$ and pain attacks in the upper abdomen. The reason for the hypoglycaemic attacks was a pancreatic tumour with already widespread liver metastases. The tumour proved to be active. Insulin levels $(104 \mu \mathrm{E} / \mathrm{ml}$; normal 1-20 $\mu \mathrm{E} / \mathrm{ml}$ ), C-peptide concentration (69 $\mathrm{ng} / \mathrm{ml}$; normal $0.6-5.4$ $\mathrm{ng} / \mathrm{ml})$, serum glucose levels $(150-250 \mathrm{mg} / 100 \mathrm{ml})$ and growth hor- mone levels $(85.9 \mathrm{ng} / \mathrm{ml}$; normal $<1 \mathrm{ng} / \mathrm{ml})$ were elevated. In the same month the pancreatic tail with an islet cell carcinoma and the left liver lobe were resected, demonstrating widespread metastases in the liver. After 8 cycles of a 5-fluorouracil and streptozocin treatment, computed tomography scans demonstrated a small decrease in the size of the liver metastases. In March 1990 an ultrasound-guided liver biopsy, examined in the laboratory of Dr. Reubi demonstrated in more than $10 \%$ of all tumor cells highly positive somatostatin receptors. A Sandostatin treatment was started with $3 \times 100 \mu \mathrm{g}$ subcutaneously per day. In April 1990 an iodine 123 tyr-3-octretide scan was performed, which demonstrated only a faint, patchy, increased uptake in the liver (Fig. 2a), which could only be interpreted as positive after knowledge of the sonographic and computed tomographic results. The scan revealed a significant pathological uptake in the right acetabulum (Fig. 2b), which was suspected to be metastatic. X-radiographs demonstrated an osteolytic process in this region. The symptoms of hygolycaemia and the patient's general condition seriously worsened, and the patient started to complain of right-sided hip pain. In June 1990 a percutaneous radiation treatment of the right hip with $10 \mathrm{MV}$ photons was started, up to a total dose of $36 \mathrm{~Gy}$. A subsequent total hip replacement (Aug. 1990) only demonstrated osteonecrosis from the radiation therapy and no tumour tissue. Despite continuing somatostatin treatment and a permanent glucose infusion, the patient died in Jan. 1991.

\section{Discussion}

Previous papers (Bloom and Polak 1980; Stefani et al. 1974) have already demonstrated that, with the exception of insulinomas, most endocrine pancreatic tumours are malignant and have metastasized at the time of diagnosis. Case report 1 describes one of these rare, infiltrating, unresectable, endocrine-inactive islet cell tumours at the time of diagnosis. Moreover, this case demonstrates very well the finding of earlier reports (Lamberts et al. $1990 \mathrm{a}, \mathrm{b}, \mathrm{c})$ that the results of gamma-camera scans after injection of iodine 123 tyr-3-octreotide, a cheap and harmless technique, have a close relationship with the in vitro detection of somatostatin receptors in the tumours using autoradiography. This indicates, therefore, that the ligands binding to the tumour in vivo indeed represent binding to specific somatostatin receptors. The fact is proven in case report 1 by the therapeutic effectiveness of octreotide via growth inhibition of this tumour over years. This points out the high specificity of this new technique of tumor imaging. Furthermore, this technique provides increased diagnostic accuracy in the presurgical localisation of insulinomas, which ranges at the moment, dependent on the method used and the localisation of the tumour, between $14 \%$ and $100 \%$ (Junginger et al. 1990).

Because there is a wide variation in the clinical presentation of endocrine pancreatic tumours in man, there are tumours which present with signs and symptoms related to the hormonal hypersecretion from these tumours, others with symptoms related to the tumour mass itself. In these latter cases, as demonstrated in case report 1 , these often undifferentiated tumours do not 


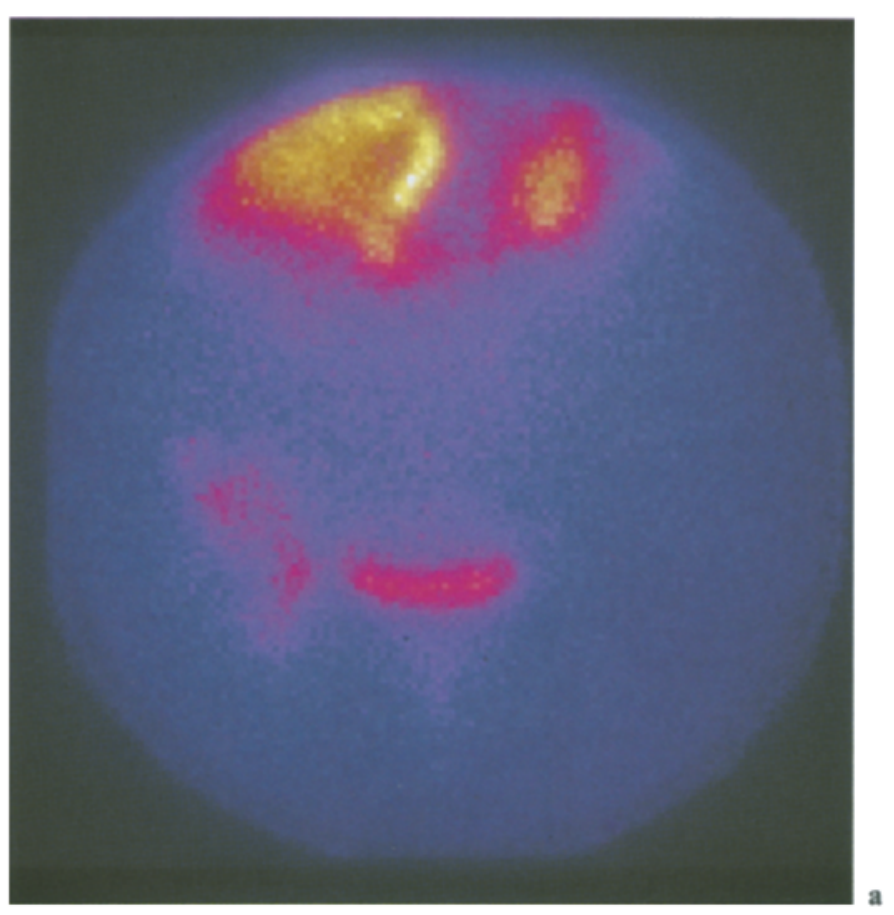

Fig. 2a. The iodine 123 tyr-3-octreotide planar scan demonstrates significant pathological uptake in the right acetabulum, where $\mathrm{x}$ radiographs later demonstrated an osteolytic tumour (anterior view). b A 60-year-old woman with metastatic somatostatin receptor-positive pancreatic islet cell carcinoma. The iodine 123 tyr-3octreotide single photon emission tomogram of the liver demonstrates a patchy, increased uptake in the metastases

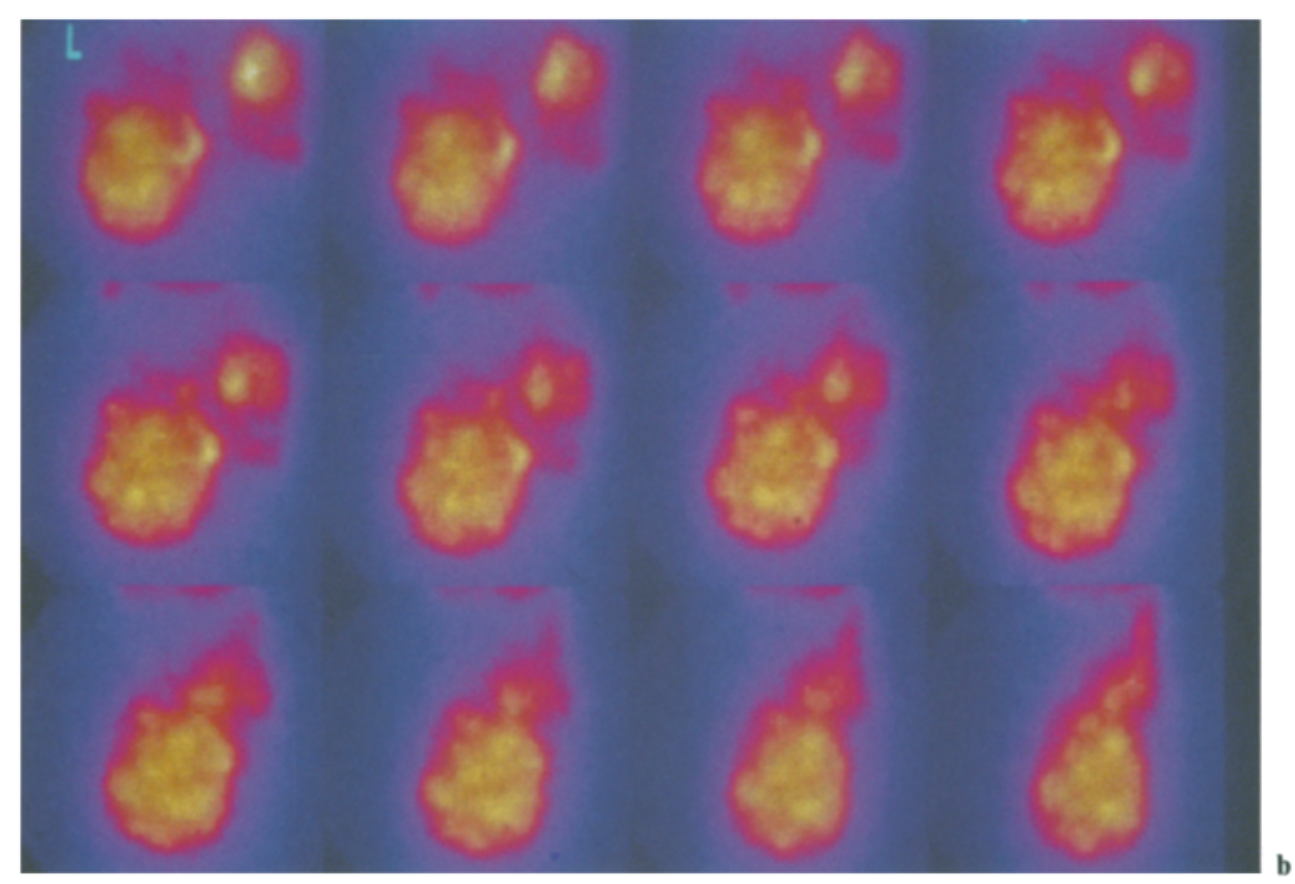

secrete considerable amounts of biologically active hormones, or the clinical picture is rather inconspicious (Lamberts et al. 1990 b; Stefani et al. 1974). Here the iodine 123 tyr-3-octreotide scan might be helpful in demonstrating somatostatin receptors on pancreatic tumours, which helps in the differential diagnosis of ductal adenocarcinomas, which have no somatostatin receptors from endocrine pancreatic carcinomas (Lamberts et al. $1990 \mathrm{c}$ ). Therefore, this method might be a safe alternative to in vivo octreotide application in the selection of insulinoma patients for chronic treatment with the drug, which has been previously proposed.
In the second patient, somatostatin receptors were shown earlier on the primary tumour. This was the indication for the somatostatin scan, because such specific receptors could be expected in the metastases also. For this reason, the known liver metastases could be detected as small areas of increased uptake in the liver, which were the reason for the increased insulin secretion in this patient. The scan demonstrated another more striking lesion in the right hip, which was found to be another metastasis from the insulinoma.

The benefit of the scan can be easily demonstrated in case report 2 , as besides the already known liver me- 
tastases another large focus was detected in the right hip before clinical symptoms appeared, leading to surgical treatment. However, due to the liver metastases the hyperinsulinaemia persisted even under continuing octreotide medication.

In conclusion, we have confirmed in this study the results of iodine 123 tyr-3-octreotide scans from other groups (Lamberts et al. 1990a). This method opens for the first time up the possibility of the localization of somatostatin receptor-positive tumours and their metastases. Second, this method offers a non-invasive procedure to determine tumour receptor status. Theoretically, this new approach of scintigraphic tumour detection might be extended to other receptor-containing tumours and by this way may offer an alternative to immunoscintigraphy with monoclonal antibodies.

Acknowledgements. We gratefully thank Dr. J. Pless (Pharma Division, Sandoz AG, Basel, Switzerland) for the support with tyr-3octreotide and Dr. J.C. Reubi (Sandoz Research Institute, Bern Switzerland) for demonstrating the somatostatin receptors in the tumour tissue

\section{References}

Bakker WH, Krenning EP, Breeman WA, Koper JW, Kooij PP, Reubi JC, Klijn JG, Visser TJ, Docter R, Lamberts SW (1990) Receptor scintigraphy with a radioiodinated somatostatin ana- logue: radiolabeling, purification, biologic activity and in vivo application in animals. J Nucl Med 31:1501-1509

Bloom SR, Polak JM (1980) Glucagonomas, VIPomas and somatostatomas. Clin Endocrinol Metab 9:285-297

Junginger T, Böttger T, Beyer J, Thelen M (1990) Der organische Hyperinsulinismus-Diagnostik und Therapie. Dtsch Ärtzebl 87: B2418-B2423

Krenning EP, Bakker WH, Breeman WAP, Koper JW, Kooij PPM, Ausema L, Lameris JS, Reubi JC, Lamberts SWJ (1989) Localization of endocrine-related tumours with radioiodinated analogue of somatostatin. Lancet I : $242-244$

Lamberts SWJ, Bakker WH, Reubi JC, Krenning EP (1990a) Treatment with sandostatin and in vivo localization of tumors with radiolabeled somatostatin analogs. Metabolism 39:152155

Lamberts SWJ, Bakker WH, Reubi JC, Krenning EP (1990b) Somatostatin-receptor imaging in the localization of endocrine tumors. N Engl J Med 323:1246-1249

Lamberts SWJ, Hofland LJ, Koetsfled van PM, Reubi JC, Bruining HA, Bakker WH, Krenning EP (1990c) Parallel in vivo and in vitro detection of functional somatostatin receptors in human endocrine pancreatic tumours: consequences with regard to diagnosis, localization and therapy. J Clin Endocrinol Metab $71: 566-574$

Reubi J-C, Hacki WH, Lamberts SWJ (1987) Hormone producing gastrointestinal tumors contain a high density of somatostatin receptors. J Clin Endocrinol Metab 65:1127-1134

Reubi JC, Kvols L, Krenning E, Lamberts SWJ (1990) Distribution of somatostatin receptors in normal and tumor tissue. Metabolism 39:78-81

Stefani P, Carboni M, Patrassi N, Basoli A (1974) Beta-islet tumors of the pancreas: results on 1067 cases. Surgery 75:597-609 